\title{
High Average Power, High Energy, Femto-second Fiber Chirped Pulse Amplification System
}

\author{
F. He, J. H. V. Price, A. Malinowski, A. Piper, M. Ibsen, D. J. Richardson \\ Optoelectronics Research Centre, University of Southampton, Southampton, SO17 1BJ, UK \\ email: feh@orc.soton.ac.uk \\ J. W. Dawson, C. W. Siders, J. A. Britten, C. P. J. Barty \\ Lawrence Livermore National Laboratory, 700 East Avenue, Livermore, CA 94550, USA
}

\begin{abstract}
We have demonstrated an Yb-fiber laser system incorporating a CFBG stretcher, bandwidth optimised amplifiers and dielectric grating compressor. The system produced $135 \mathrm{~W}$ average power with pulse energy of $13.5 \mu \mathrm{J}$. The recompressed pulse duration was $360 \mathrm{fs}$. (C) 2007 Optical Society of America

OCIS codes: (140.3510) Lasers, fibers; (140.7090) Ultrafast lasers; (060.4370) Nonlinear optics, fiber
\end{abstract}

\section{Introduction}

Applications requiring femtosecond pulses with both high average powers and high pulse energies range from industrial materials processing to high-harmonic and plasma-based x-ray generation. Fiber systems can be diodepumped, high gain, compact and robust, and at high powers they typically suffer less from thermo-optical problems when compared to bulk crystal and glass laser sources. However, nonlinear effects in the fiber amplifiers are a key limitation for pulsed fiber systems and further technological developments are therefore required. Here we report an $\mathrm{Yb}$-fiber femto-second chirped pulse amplification (CPA) system incorporating several novel technologies which produces high-quality, high-energy pulses, at high average power.

Critical components of our system are a chirped-fiber-Bragg-grating (CFBG) stretcher designed with both $2^{\text {nd }}$ and $3^{\text {rd }}$ order dispersion compensation matched to the compressor [1], an Yb-fiber amplifier chain with bandwidth optimized using modelling tools [2], and a dielectric grating based compressor which has the advantages of high power handling compared to gold gratings without the size constraints associated with fused silica transmission gratings. The system produced $135 \mathrm{~W}$ after the amplifier chain at $10 \mathrm{MHz}$ pulse repetition rate $(13.5 \mu \mathrm{J}$ pulse energy) and the recompressed pulse duration was $360 \mathrm{fs}$. To our knowledge this is the highest combined average power and pulse energy when compared to either high average power systems with lower pulse energies [3], or high pulse energy systems with lower average powers [4].

\section{Experimental setup}
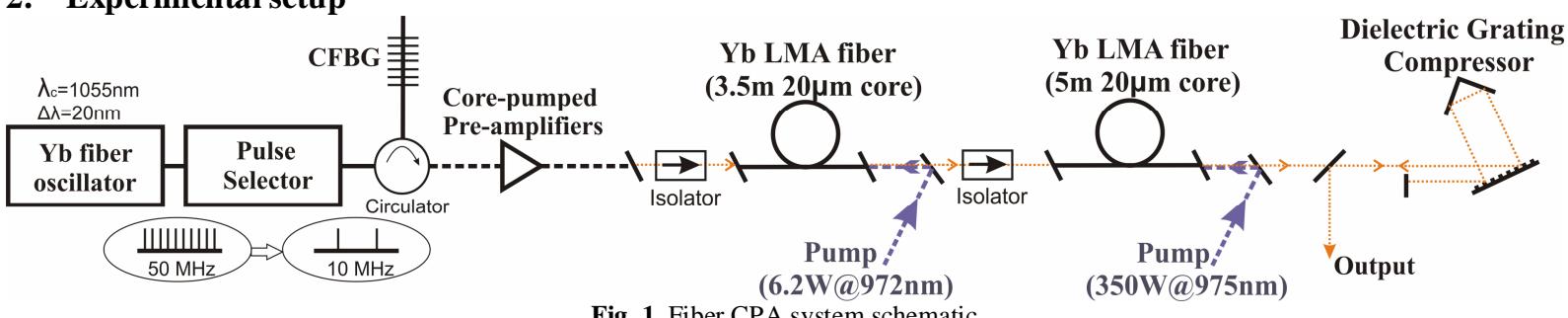

Fig. 1. Fiber CPA system schematic

The schematic of our Yb fiber CPA system is shown in Fig. 1. The passively mode-locked diode-pumped Yb-fiber seed laser provided < 150 fs pulses with $\sim 20 \mathrm{~nm}$ bandwidth centred at $1055 \mathrm{~nm}$ [2]. An Electro-Optic Modulator with $>40 \mathrm{~dB}$ extinction ratio was used to reduce the pulse repetition rate from $50 \mathrm{MHz}$ to $10 \mathrm{MHz}$. The pulses were then coupled into a CFBG using a fiberised circulator and were stretched to $\sim 2$ ns duration. Core-pumped preamplifiers increased the power to $\sim 0.1 \mathrm{~W}$ before launching the pulses into the high power cladding-pumped amplifiers (core diameter $20 \mu \mathrm{m}$, NA 0.06; inner cladding diameter $200 \mu \mathrm{m}$, NA 0.5). Isolators, wave-plates and accousto-optic modulators were incorporated between the amplifiers to prevent ASE build-up. The final amplifier used $5 \mathrm{~m}$ of fiber and was pumped by a $975 \mathrm{~nm}, 350 \mathrm{~W}$ laser diode. A dielectric grating was then used to recompress the pulses at the output.

\section{Results}

We first characterised the CFBG stretcher and final high power Yb fiber amplifier. The performance of the CFBG in the orientation used in the CPA system is shown in Fig.2.a). The reflected spectrum has $\sim 12 \mathrm{~nm}$ bandwidth, only $\sim 2.5 \mathrm{~dB}$ reflectivity variation and minimal spectral ripple $(0.01 \mathrm{~nm}$ resolution $)$. The measured dispersion was obtained using the phase delay measurement technique with a tunable laser source, and it is very close to the 
specification. Fig. 2.b) shows the output power after the final amplifier. The maximum power of $135 \mathrm{~W}$ corresponds to a slope efficiency of $77 \%$ with respect to the launched pump power.

(a) CFBG Characteristics

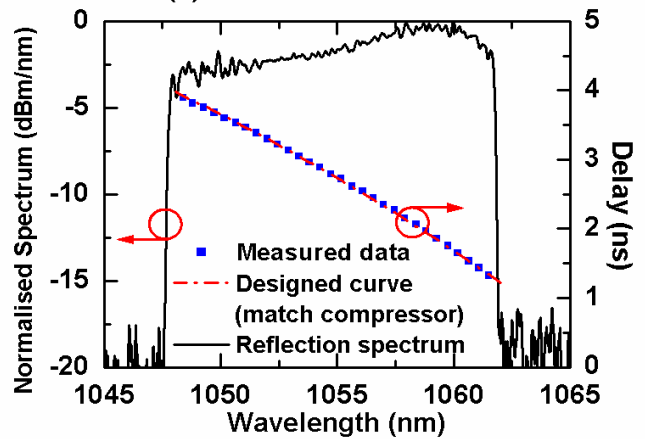

(b)

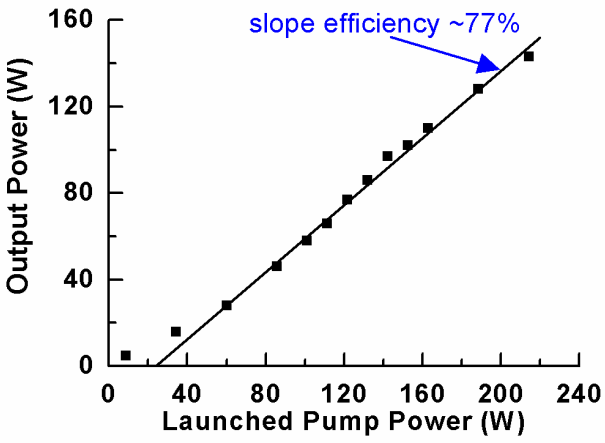

Fig. 2. a) Experimentally measured CFBG characteristics. b) Output power characterization of the final Yb-fiber amplifier in the CPA system

The temporal and spectral characteristics of the pulses are shown in Fig.3. Fig.3.a) shows the autocorrelation traces at different pulse energies. The autocorrelation width remained constant at $510 \mathrm{fs}$, corresponding to a pulse width of 360 fs assuming a Gaussian shape $(\Delta v \Delta \tau \sim 0.6)$. Due to SPM in the final amplifier, the pedestal was observed to increase at a pulse energy of $13.5 \mu \mathrm{J}$, but the majority of the energy remained in the central peak. Measurements at reduced repetition rates showed more severe pulse distortions at a pulse energy of $20 \mu \mathrm{J}$, and stimulated Raman scatting at a pulse energy of $\sim 200 \mu \mathrm{J}$. The measured compressor transmission efficiency of $52 \%$ indicates a maximum compressed output pulse energy of $7 \mu \mathrm{J}$ with average power of $70 \mathrm{~W}$. Fig.3.b) shows that the $12 \mathrm{~nm}$ bandwidth after the CFBG stretcher is maintained though the amplifier chain. There is no spectral distortion at $135 \mathrm{~W}$ output power and the signal remains $20 \mathrm{~dB}$ above the ASE level. The compressed pulse spectrum had a bandwidth of $6.2 \mathrm{~nm}$, limited by size of some of the mirrors, and we are currently working to improve the compressor performance.

(a)

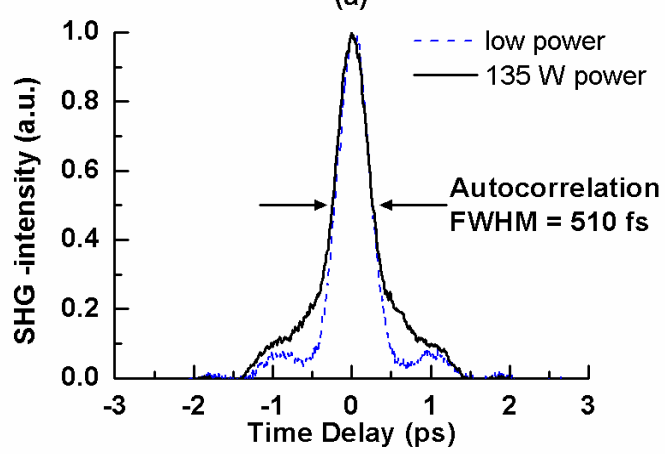

(b)

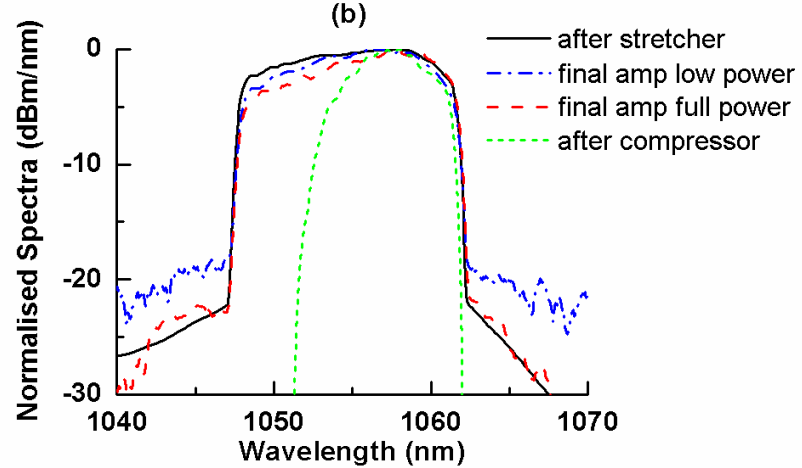

Fig. 3. Output characteristics of fiber CPA system. a) autocorrelation traces at low and high pulse energies. b) spectra of the pulses before and after the grating compressor at $135 \mathrm{~W}$ output power.

\section{Conclusion}

We have demonstrated an Yb-fiber chirped pulse amplification system incorporating a CFBG stretcher, optimised bandwidth amplifiers and a dielectric grating compressor. The system produced $135 \mathrm{~W}$ average power with pulse energy of $13.5 \mu \mathrm{J}$. The recompressed pulse duration was $360 \mathrm{fs}$. We believe that this demonstration of high pulse energies at high average powers will further broaden the applications of femto-second fiber-based CPA systems.

\section{References}

[1] J. H. V.Price, et Al., "Advances in high power short pulse fiber laser systems and technology," in Proceedings of SPIE, Vol. 5709 Fiber Lasers II: Technology, Systems, and Applications, (The International Society of Optical Engineering, 2005), pp.184-192.

[2] F. He, et Al., "Optimisation of short pulse multi-stage Yb fiber amplifier systems using commercial gain-modelling software," in Conference on Lasers and Electro-optics, (Optical Society of America, 2006), paper CThR6.

[3] J. Limpert, F. Roser, et Al. , "High-power ultrafast fiber laser systems," IEEE J. Sel. Topics Quantum Electron. 12, 233-244 (2006).

[4] A. Galvanauskas, "Mode-scalable fiber-based chirped pulse amplification systems," IEEE J. Sel. Top. Quant. Electron. 7, 504-517 (2001). 